\title{
Evaluation of Treatments in Patients with Nocturnal Bruxism on Bite Force and Occlusal Contact Area: A preliminary report
}

\author{
Alper Alkan \\ Emel Bulut ${ }^{\mathrm{b}}$ \\ Selim Aricic \\ Shuichi Sato ${ }^{d}$
}

Objectives: The purpose of this study was to investigate the short-term effects of occlusal splint therapy and tricyclic antidepressants on the bite force and occlusal contact area of patients presenting with nocturnal bruxism.

Methods: A maxillary full-coverage hard acrylic splint was applied to the five patients (Group S). Five patients took a tricyclic antidepressant (Amitriptiline $\mathrm{HCl}, 10 \mathrm{mg} /$ day) for 3 months (Group A) and a control group (Group C) comprising of 10 dental school students with normal occlusion was also formed. Using a Dental Prescale (Fuji Photo Film Corporation, Tokyo, Japan) and an Occluzer computer (FPD703, Fuji Photo Film Corporation, Tokyo, Japan) the bite force and occlusal contact area were measured. The evaluations were made just before the treatment and at 1 month and 3 months of treatment.

Results: The bite force and occlusal contact area before treatment in study Groups A and S were found to be higher than those in the Group C. Furthermore, the bite force and occlusal contact area increased during treatment in Group A whilst they decreased in Group S. Bite force and occlusal contact area in Group S were lower at both 1 month and 3 months of treatment than in Group C.

Conclusions: It could be tentatively suggested that occlusal splint therapy may be more effective than tricyclic antidepressant in the treatment of bruxism. Further investigations of this measurement method involving larger study populations and a longer follow-up period are needed. (Eur J Dent 2008;2:276-2821

Key words: Antidepressant; Bite force; Bruxism; Occlusal contact area; Splint.

\footnotetext{
Associate Professor and Chairman, DDS, PhD, Department of Oral and Maxillofacial Surgery, Faculty of Dentistry, Erciyes University, Kayseri, Turkey.

b Assistant Professor, DDS, PhD, Department of Oral and Maxillofacial Surgery, Faculty of Dentistry, Ondokuz Mayis University, Samsun, Turkey. Professor, DDS, MMedSci, PhD, Department of Orthodonthics, Faculty of Dentistry, Ondokuz Mayis University, Samsun, Turkey.
}

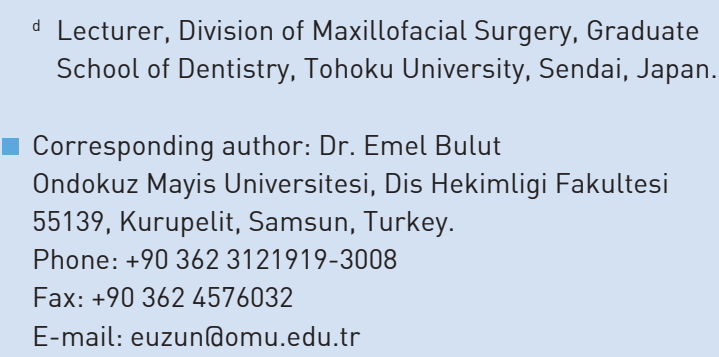




\section{INTRODUCTION}

The Glossary of Prosthodontic Terms defines bruxism as the parafunctional grinding of teeth or an oral habit consisting of involuntary rhythmic or spasmodic nonfunctional gnashing, grinding, or clenching of teeth in other than chewing movements of the mandible, which may lead to occlusal trauma. ${ }^{1}$ Parafunctional masticatory activity includes nonproductive diurnal and nocturnal clenching or grinding of teeth (bruxism), which is associated with stress and is generally believed to have deleterious effects on the masticatory system. ${ }^{2}$

Patients report such activities during sleep and sometimes even more whilst awake. Psychological factors such as anxiety or stress increase the rate of bruxism. ${ }^{3}$ Parafunctional activities can also produce muscle pain. ${ }^{4} \mathrm{~A}$ more complex variant of the "bruxing leads to pain" model, the psychophysiological model of myofascial face pain, postulates that stress is an initiating and maintaining cause of bruxism. That is, life stressors are believed to lead to distress or "tension" in the individual, which can then lead to dysfunctional oral habits, tooth-grinding or clenching. ${ }^{5}$

The treatment of bruxism has involved several different techniques: (i) reversible occlusal therapy, such as various occlusal appliances; (ii) emotional stress therapy, such as relaxation techniques, biofeedback, and hypnotherapy; (iii) physiotherapy, such as Transcutaneous Electrical Nerve Stimulation, acupuncture, and manual techniques; and (iv) pharmacologic therapy, such as nonopioid analgesics, corticosteroids, anxiolytic agents, local anestetics, tranquilizers, muscle relaxants, and tricyclic antidepressants (TCAs). TCAs have been specifically advocated for the treatment of nocturnal bruxism. ${ }^{6}$

In clinical research on occlusion, various techniques are used to assess the nature of occlusal contacts in the intercuspal position. However, few techniques can make a quantitative analysis of occlusal contact area and occlusal force. Recently, a pressure-sensitive device, the Dental Prescale \& Occluzer computer, was developed by Fuji Photo Film Co (Tokyo, Japan). ${ }^{7}$

The aim of this preliminary study was to evaluate the short-term effects of maxillary fullcoverage occlusal splint therapy and the use of
TCA on bite force and occlusal contact area in patients with nocturnal bruxism. Comparison of the results with those of the healthy subjects was also done.

\section{MATERIALS AND METHODS}

Ten patients ( 9 females, 1 male) with a history of nocturnal bruxism participated in this study. The mean age of the patients was 20.5 years (range, 18-29 years). Clinical examinations were completed intraorally and extraorally by the same examiner (E.B). The intraoral examination included maximum mouth opening and an occlusal examination. The mean of their maximum mouth opening was $40.12 \pm 6.5$ millimeter. The pain intensity was continuously scored by the subjects on a $10 \mathrm{~cm}$ visual analogue scale (VAS) with the lower extreme labeled 'no pain' and the upper extreme labeled 'most pain imaginable'. The outcome measures of VAS was taken at baseline, at 1 months and at 3 months after the start of treatment.

The extraoral examination included the temporomandibular joint (TMJ) and muscle examination. The inclusion criteria for this study group were:

1. The presence of nocturnal clenching or grinding of teeth.

2. The presence of dull, continuous pain localized in a masticatory muscle area.

3. Patients experienced pain in the morning.

4. No complaints of symptoms of temporomandibular joint (TMJ) disorders.

5. All of the study subjects had to have complete dentitions with Class I molar relationships.

Consent forms were obtained from all subjects and the study was approved by the Ethical Committee for Ondokuz Mayis University. The bruxing patients were randomly divided into two groups for splint or amitriptiline therapy applications. The same examiner delivered both the splint and medication therapies (E.B). The bite force and occlusal contact area were checked by a second examiner (S.S) who was not informed of the therapy that each individual was receiving.

A maxillary full-coverage hard acrylic appliance, which is often called a stabilization splint, was used in five patients (Group S). The thickness of the splint was approximately one 
millimeter at the posterior region, just enough to release the occlusal contact between the upper and lower teeth. The appliance was adjusted on every visit to maintain a stable contact relationship with each opposing mandibular tooth with no exclusive guidance in the position of relaxed closure from a postural rest position. The patients were instructed to wear the appliance full time except when eating or brushing their teeth. Five patients were treated with TCA (Amitriptiline $\mathrm{HCl}, 10 \mathrm{mg} /$ dayl (Group A).

The bite force and occlusal contact area measurements were recorded pre-treatment and at 1 month and 3 months of treatment. All measurements were examined using a pressuresensitive sheet (Dental Prescale, 50 H, type R, 97 $\mu \mathrm{m}$ thick) and its analyzing apparatus (Occluzer) (Figure 1). When the sheet is bitten, microcapsules containing a color forming material are broken to react with a color-developing material. The occlusal contact is detected by this chemical reaction and scanned by the occlusion pressure graph. Bite force (Newton) and occlusal contact area $\left(\mathrm{mm}^{2}\right)$ are calculated from the degree of coloring $^{7-9}$ (Figure 2). During measurement, the subjects were seated with their heads in an unsupported natural position, looking forward. A Dental Prescale was placed between the upper and lower dental arch, and the subjects were instructed to bite as forcefully as possible for about 3 seconds.

A control group (Group C) comprising 10 dental school students with normal occlusion (7 women, 3 men; mean age 20.5 years) was also formed in order to compare the bite force and occlusal contact area measurements with those of the study groups. They did not have a history of bruxism, and were not receiving any treatment.

\section{Statistical analysis}

In this study, a general linear model (repeated measures) analysis was used to investigate the effects of the time (pre-treatment, 1 month and 3 months of treatment) on the bite force and occlusal contact area. This analysis showed that time had statistically significant effects on the bite force and occlusal contact area for each group (Group A and Group S). A nonparametric test (Wilcoxon) was also used to test the hypothesis that there were no significant differences between the three subgroups (pre-treatment, 1 month and 3 months of treatment) of the two study groups for the mean bite force and occlusal contact area assessments. To compare the bite force and occlusal contact area between the study and control groups, another nonparametric test (Kruskall Wallis) was also used. Nonparametric statistical analyses were chosen due to the limited number of the samples. All statistical analyses were accomplished by using the Statistical Package for the Social Sciences (Version 12.0, SPSS Inc., Chicago, III, USA) at the 95\% confidence level $(P<.05)$.

\section{RESULTS}

Table 1 shows the descriptive statistics for the groups. Using the occlusal contact area or bite force as the dependent variable, general linear model analysis showed significant differences between Groups A and S; $P=0.016$ and $P=0.018$, respectively.

Furthermore, mean values of the occlusal contact area and bite force of the subgroups (pretreatment, 1 month and 3 months of treatment) showed statistically significant differences between Group A and Group S.

There were significant differences in the occlusal contact area and bite force between pretreatment and at 1 month of treatment, and pretreatment and at 3 months of treatment in Group $A$ $(P<.05)$. It was observed that the occlusal contact area and bite force generally increased with time in Group A, with the exception of the bite force at 3 months of treatment. On the contrary, it was observed that the occlusal contact area and bite force decreased with treatment in Group S. There were significant differences in the bite force and occlusal contact area between pre-treatment and at 3 months of treatment in Group $S(P<.05)$ (Figures 3 and 4).

The bite force and occlusal contact area at pre-treatment in the study groups were found to be higher than those in Group C. The bite force and occlusal contact area in Group $S$ at 1 month and at 3 months of treatment were lower than those in Group C. However, there were statistically significant differences at 3 months of treatment $(P<.05)$. On the contrary, bite force and occlusal contact area at 1 month and 3 months of treatment in Group A were found to be greater 
than those in Group C.

Figures 5 and 6 show the VAS scores of the groups.

\section{DISCUSSION}

In clinical research on occlusion, various techniques are used to assess the nature of occlusal contact in the intercuspal position. Usually, this is measured with a custom bite transducer or T-Scan system. ${ }^{10-13}$ It has been suggested that these measurement systems occasionally misread the occlusal contact area and bite force because they are fairly inflexible or thick. ${ }^{8}$ Recently, a pressure sensitive device that is very thin and flexible has been approved for dental use. Bite force, occlusal contact area, and occlusal balance are measured and analyzed using this device. When these advantages are

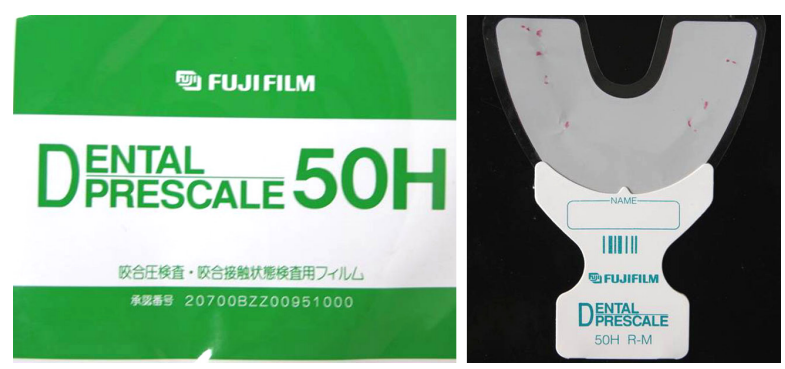

Figure 1. Dental Prescale.

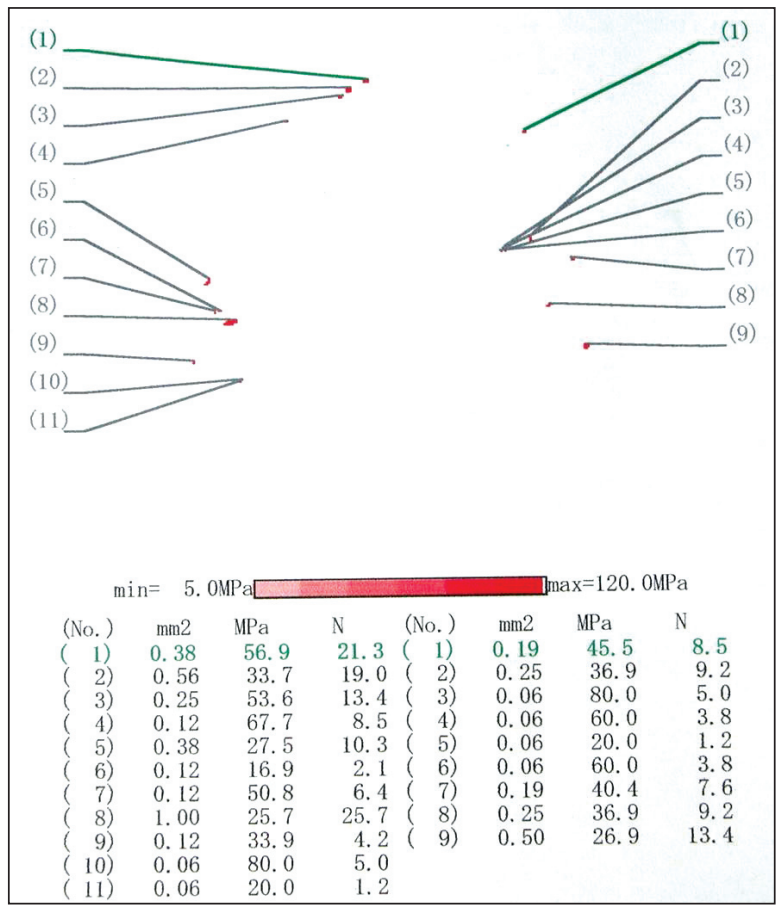

Figure 2. Analysis of biting pressure sensitive sheet bite force $(\mathrm{BF})$, bite pressure (BP) and occlusal contact area (OCA). BF (newtons [N]): Pressure multiplied by the area. OCA (square millimeters): Total area of the impressed marks on the sheet. BP (megapascals [MPa]): The BF per $1 \mathrm{~mm}^{2}$ of the OCA. considered, we can tentatively suggest that the Dental Prescale is a suitable tool for the assessment of occlusal contact area and bite force.

Measurement with the pressure-sensitive sheet is affected not only by the activity of masticatory muscles but also by dental and occlusal conditions. ${ }^{8}$ Recently, Miyaura et $\mathrm{l}^{14}$ used a pressure detecting sheet in an epidemiological study to evaluate the biting abilities of each participant. As a result, the biting abilities were closely correlated with the number of present teeth. ${ }^{15}$ Therefore; we selected patients that had complete dentitions in this study.

The literature has numerous reports implicating parafunctional activity as having a significant role in the cause of temporomandibular disorders (TMD). ${ }^{2,16}$ Capurso ${ }^{17}$ collected data

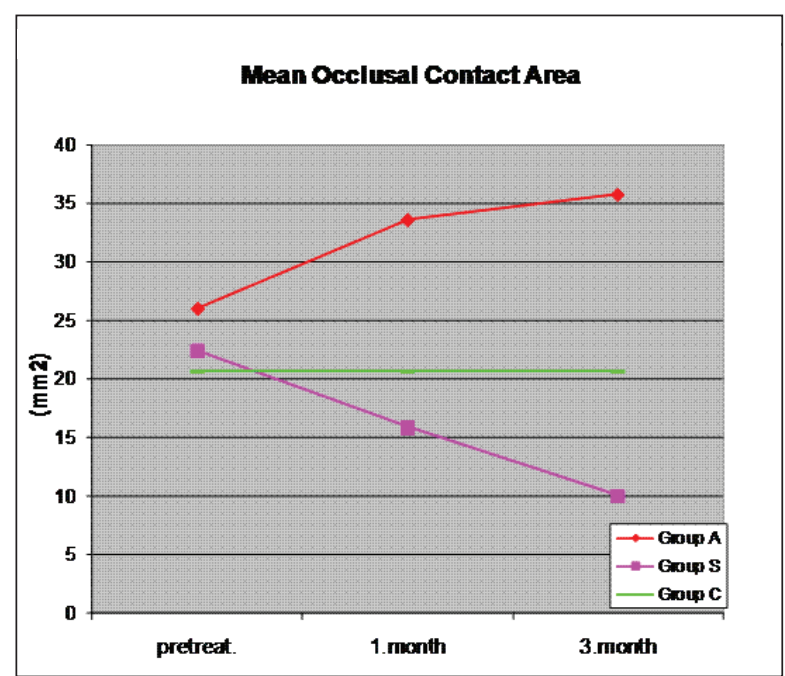

Figure 3. Changes in the mean occlusal contact area in study groups and control.

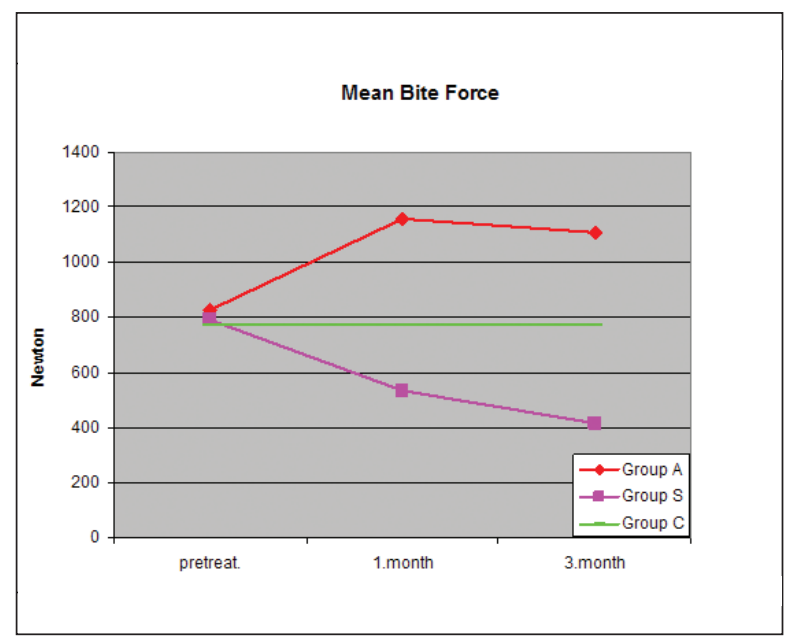

Figure 4. Changes in the mean bite force in study groups and control. 
from 406 patients with signs and symptoms of craniomandibular disorders and concluded that bruxism, present in $35.9 \%$ of his patient population, was a major causative factor. Rugh and Harlan ${ }^{18}$ reviewed the effects of nocturnal bruxism on TMDs and concluded that this habit can cause significant damage to every part of the masticatory apparatus. Patients complaining of any symptoms of temporomandibular joint (TMJ) disorders were not included in this study because many conditions can produce signs and symptoms similar to those of temporomandibular joint (TMJ) disorders.

Solberg et $\mathrm{al}^{19}$ reported that nocturnal masseteric muscle activity, as measured by electromyography, was reduced immediatelyafter the insertion of a full arch maxillary stabilization splint. Lobbezoo et $\mathrm{al}^{20}$ and Visser et $\mathrm{al}^{21}$ reported a decrease in temporal muscle activity after the insertion of the stabilization splint. It was also reported that the stabilization splint caused improvement in the masseter muscular activity balance. Kurita et $\mathrm{al}^{22}$ suggested that the use of the stabilization splint has the effect of reducing hyperactivity and asymmetry in the activity of the jaw elevator muscles, and consequently brings a stable and physiologically optimal occlusal force from the muscles. They suggested that the decreased masticatory muscle activity with the use of the splint was responsible for the decrease in the area of each occlusion point.

In the light of this data, it may be possible to comment on muscle activity regarding the changes in occlusal contact area and bite force. Numerous studies have been conducted on the changes in muscle activity of bruxism patients. However, these studies may be interpreted as impractical due to time wasted during these studies and the equipment requirements. Therefore, this study has been conducted using a "Dental Prescale" which may produce extremely practical and objective results.

In our study, we have observed that the occlusal contact area and bite force decreased in patients

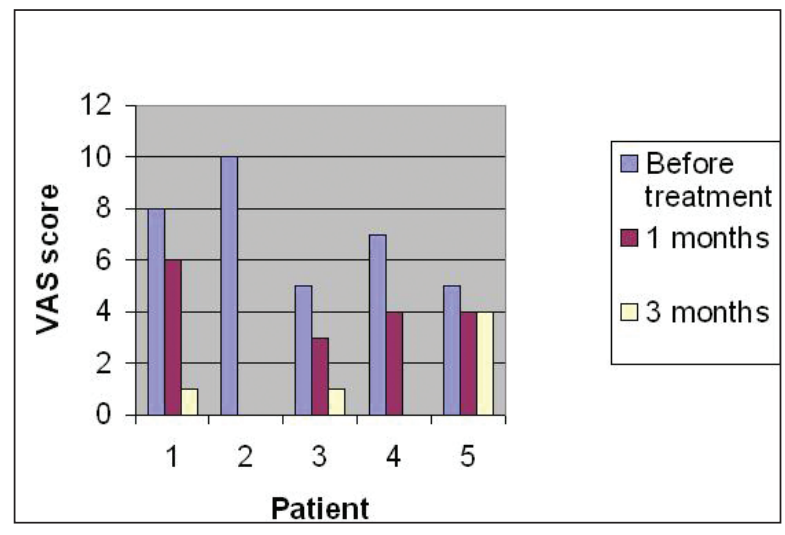

Figure 5. VAS scores of group S.

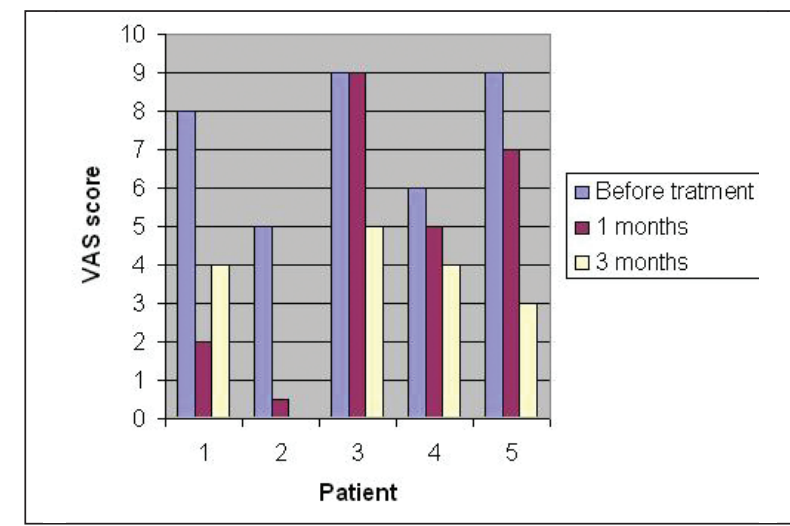

Figure 6. VAS scores of group A.

Table 3. Mean bite force (newton) and occlusal contact area (square millimeter) of the patients and control group.

\begin{tabular}{|c|c|c|c|c|c|c|}
\hline \multirow[t]{2}{*}{ Groups } & \multirow[t]{2}{*}{$\mathrm{N}$} & \multirow[t]{2}{*}{ Time } & \multicolumn{2}{|c|}{ Bite force (Newton) } & \multicolumn{2}{|c|}{$\begin{array}{l}\text { Occlusal contact area } \\
\qquad\left(\mathrm{mm}^{2}\right)\end{array}$} \\
\hline & & & Mean & SD & Mean & SD \\
\hline \multirow[t]{3}{*}{ Group A } & 5 & BT & 827.00 & 620.89 & 26.04 & 17.82 \\
\hline & & 1 month & 1158.22 & 566.10 & 33.63 & 15.45 \\
\hline & & 3 months & 1106.58 & 630.29 & 35.80 & 17.53 \\
\hline \multirow[t]{3}{*}{ Group S } & 5 & BT & 793.82 & 352.16 & 22.44 & 11.17 \\
\hline & & 1 month & 533.30 & 288.31 & 15.89 & 8.05 \\
\hline & & 3 months & 413.46 & 99.64 & 10.08 & 3.40 \\
\hline Group C & 10 & & 773.24 & 194.44 & 20.75 & 5.98 \\
\hline
\end{tabular}

BT: Before Treatment, SD: Standart Deviation 
using a splint by the end of the third month and that these values increased in patients using tricyclic antidepressants. In fact, the increase in the occlusal contact area and bite force after a prosthodontic, orthodontic or surgical treatment is a desired and expected outcome. However, the purpose of treatment in bruxism patients is to reduce the high muscle activity by using antidepressants and/or a splint, thereby relieving the pain by reducing the occlusal contact area and bite force. In our study, the bite force and occlusal contact area in Group $S$ at 1 month and 3 months of treatment were lower than those in the Group C. We have concluded that the splint treatment is superior to tricyclic antidepressants in bruxism patients. We have also observed a difference between groups with regard to pain values indicated on a VAS. We detected a decrease in pain in both groups. However, the VAS scores of Group S were higher than Group A. The literature reports that occlusal balance and muscle function are significantly correlated. ${ }^{23}$

TCAs have been specifically advocated for the treatment of nocturnal bruxism. However, only a few randomized clinical controlled studies have tested the usefulness and the efficacy of TCAs in managing the signs and symptoms of nocturnal bruxism. ${ }^{6,24}$ Their low-abuse potential, known side effects, analgesic characteristics that are separate from their antidepressant qualities, and relatively low cost have all contributed to the decision to use them. ${ }^{25}$ Amitriptyline is effective in the treatment of chronic oral-facial pain and that its efficacy is independent of its effects on depression. It appears that tricyclic antidepressants act in a different fashion from opiate drugs that alter the sensory discriminative component of pain. ${ }^{6}$ Raigrodski et al ${ }^{25,26}$ reported that results of their study do not support the administration of small doses of amitriptyline over a period of 4 weeks for the management of pain resulting from sleep bruxism. However, the results support the administration of small doses of amitriptyline ( $25 \mathrm{mg} / \mathrm{night}$ ) for the management of the perception of stress levels associated with sleep bruxism. Mohamed et $\mathrm{al}^{24}$ reported that ten adult subjects with sleep bruxism were administered amitriptyline (25 mg/night) and placebo (25 mg/night), each compound over a period of one week. Neither the intensities and locations of pain nor the nocturnal masseteric electromyographic activities were significantly affected by the tricyclic antidepressant. In fact, intake of amitriptyline was unpredictably associated with either an increase or a decrease in masseteric electromyographic activity (microV.s/ min of sleep). Based on their study, small doses of amitriptyline cannot be recommended for the control of sleep bruxism and associated discomfort. Although in our study subjects with sleep bruxism were administered amitriptyline (10 mg/night) for longer periods (3 months) than the abovementioned studies, this small dose level may give an explanation for the increase of the bite force and occlusal contact area in group A. One may consider using higher doses for longer periods to decrease the bite force in sleep-bruxism, but it should be kept in mind that higher dose applications of amitriptyline have been reported to have adverse effects. ${ }^{27}$

\section{CONCLUSIONS}

Although one of the primary requirements in designing a full-scale clinical trial is an adequate sample size, in this preliminary study the records of only ten patients with bruxism were used and the short-term effects of the two different treatment methods on occlusal contact area and bite force were examined. The results of the present study can provide new information on this issue. However, a prolonged follow up study on a larger patient population must be performed in the future. It seems to be helpful to follow occlusal changes after treatment of bruxism using a Dental Prescale. This measurement method could be used to evaluate the results of the treatment of bruxism.

\section{REFERENCES}

1. Bruxism, in: The Glossary of Prosthodontic Terms(ed7). $J$ Prosthet Dent 1999; 81:5.

2. Israel HA, Diamond B, Saed- Nejad F, Raatcliffe A. The relationship between parafunctional masticatory activity and arthroscopically diagnosed temporomandibular joint pathology. J Oral Maxillofac Surg 1999;57:1034-1039.

3. Silverman S, Eversole LR, Trulove EL. Essentials of Oral Medicine. Hamilton-London: BC Decker Inc; 2001.

4. Bowley JF, Marx DB. Masticatory muscle activity assessment and reliability of a portable electromyographic instrument. J Prosthet Dent 2001;85:252-260. 
5. Janal MN, Raphael KG, Klausner J, Teaford M. The role of tooth-grinding in the maintenance of myofascial face pain: A test of alternate models. Pain Medicine 2007;8:486-496.

6. Sharav $Y$, Singer E, Schmidt E, Dionne RA, Dubner R. The analgesic effect of amitriptyline on chronic facial pain. Pain 1987;31:199-209.

7. Sato S, Ohta M, Sawatari M, Kawamura H, Motegi K. Occlusal contact area, occlusal pressure, bite force and masticatory efficiency in patients with anterior disc displacement of the temporomandibular joint. J Oral Rehabil 1999;26:906-911.

8. Ohkura K, Harada K, Morishima S, Enomoto S. Changes in bite force and occlusal contact area after orthognathic surgery for correction of mandibular prognathism. Oral Surg Oral Med Oral Pathol Oral Radiol Endod 2001;91:141145.

9. Hidaka 0 , Iwasaki M, Saito M, Morimoto T. Influence of clenching intensity on bite force balance, occlusal contact area, and average bite pressure. J Dent Res 1999;78:13361344.

10. Ellis E, Throckmorton GS, Sinn DP. Bite forces before and after surgical correction of mandibular prognathism. $J$ Oral Maxillofac Surg 1996;54:176-181.

11. Iwase $M$, Sugimori M, Kurachi $Y$, Nagumo M. Changes in bite force and occlusal contacts in patients treated for mandibular prognathism by orthognathic surgery. J Oral Maxillofac Surg 1998;56:850-855.

12. Kim YG, Oh SH. Effects of mandibular setback surgery on occlusal force. J Oral Maxillofac Surg 1997;55:121-126.

13. Throckmorton GS, Buschang PH, Ellis E. Improvement of maximum occlusal forces after orthognathic surgery. $J$ Oral Maxillofac Surg 1996;54:1080-1086.

14. Miyaura K, Matsuka Y, Morita M, Yamashita A, Watanabe $T$. Comparison of biting forces in different age and sex groups: a study of biting efficiency with mobile and nonmobile teeth. J Oral Rehabil 1999;26:223-227.

15. Miyaura K, Morita M, Matsuka Y, Yamashita A, Watanabe T. Rehabilitation of biting abilities in patients with different types of dental prostheses. J Oral Rehabil 2000;27:10731076.

16. Allen JD, Rivera-Morales WC, Zwemer JD. Occurrence of temporomandibular disorders symptoms in healthy young adults with and without evidence of bruxism. Cranio 1990;8:312-318.

17. Capurso U. Clinical aspects of craniomandibular disorders. I. Analysis of a sample group of patients and diagnostic classification. Minerva Stomatol 1996;45:311-320.

18. Rugh JD, Harlan J. Nocturnal bruxism and temporomandibular disorders. Adv Neurol 1988;49:329341.
19. SolbergWK, ClarkGT, Rugh JD. Nocturnal electromyographic evaluation of bruxism patients undergoing short term splint therapy. J Oral Rehabil 1975;21:215-223.

20. Lobbezoo F, Van Der Glas HW, Van Kampen FM, Bosman F. The effect of an occlusal stabilization splint and the mode of visual feedback on the activity balance between jaw elevator muscles during isometric contraction. $J$ Dent Res 1993;72:876-882.

21. Visser A, Naeije M, Hansson TL. The temporal masseter coconstruction: an electromyographic and clinical evaluation of short term stabilization splint therapy in myogenous CMD patients. J Oral Rehabil 1995;22:387-389.

22. Kurita H, Ikeda K, Kurashina K. Evaluation of the effect of stabilization splint on occlusal force in patients with masticatory muscle disorders. J Oral Rehabil 2000;27:7982.

23. Ferrario VF, Serrao G, Dellavia C, Carusa E, Sforza C. Relationship between the number of occlusal contacts and masticatory muscle activity in healthy young adults. $J$ Craniomandibular Practice 2002;20:91-98.

24. Mohamed SE, Christensen LV, Penchas J. A randomized double-blind clinical trial of the effect of amitriptyline on nocturnal masseteric motor activity (sleep bruxism). Cranio 1997;15:326-332.

25. Raigrodski AJ, Mohamed SE, Gardiner DM. The effect of amitriptyline on pain intensity and perception of stress in bruxers. J Prosthodont 2001;10:73-77.

26. Raigrodski AJ, Christensen LV, Mohamed SE, Gardiner DM. The effect of four-week administration of amitriptyline on sleep bruxism. A double-blind crossover clinical study. Cranio 2001;19:21-25. Comment in: Cranio 2001;19:149.

27. McQuay HJ, Carroll D, Glynn CJ. Dose-response for analgesic effect of amitriptyline in chronic pain. Anaesthesia 1993;48:281-285. 